\title{
Ferulic acid combined with skeletal stem cells to repair irradiated bone defect
}

Jia-Wu Liang ${ }^{1,2,3,7}$,M.D., Pei-Lin Li ${ }^{1,2,7}$, M.D., Qian Wang ${ }^{1,2,3}$, M.D. \& Ph.D., Song Liao ${ }^{1,2,3}$,

M.D. \& Ph.D., Wei Hu ${ }^{1,2,3}$,M.D. \& Ph.D., Zhi-Dong Zhao ${ }^{1,2,3}$,M.D. \&Ph.D., Zhi-Ling Li ${ }^{1,2}$, M.D.,

Bo-Feng Yin ${ }^{1,2}$, M.D., Ning Mao ${ }^{4}$, M.D, Li Ding ${ }^{1,5^{* *}}$ M.D. \& Ph.D. and Heng Zhu ${ }^{1,2,4,6^{*}}$ M.D. \&

Ph.D.

1. Beijing Key Laboratory for Radiobiology, Beijing Institute of Radiation Medicine, Beijing 100850, People's Republic of China;

2. Department of Experimental Hematology \& Biochemistry, Beijing Institute of Radiation Medicine, Road Taiping 27, Beijing 100850, P.R. China;

3. People's Liberation Army General Hospital, Road Fuxing 28, Beijing, 100853, P.R. China;

4. Beijing Institute of Basic Medical Sciences, Road Taiping 27, Beijing 100850, P.R. China;

5. Air Force Medical Center, PLA, Road Fucheng 30, Beijing 100142, P.R.China;

6. Graduate School of Anhui Medical University, Road Meishan 81, Hefei 230032, Anhui, P.R., China;

7. These authors contributed equally to this work.

\section{Author Contributions}

Jia-Wu Liang: Conception and design, Collection and/or assembly of data, Data analysis and interpretation, Manuscript writing

Pei-Lin Li: Collection and/or assembly of data, Data analysis and interpretation, Manuscript writing

Qian Wang: Collection and/or assembly of data, Data analysis and interpretation,

Song Liao: Collection and/or assembly of data, Data analysis and interpretation

Wei Hu: Collection and/or assembly of data 
Zhi-Dong Zhao: Collection and/or assembly of data

Zhi-Ling Li: Collection and/or assembly of data

Bo-Feng Yin: Collection and/or assembly of data

Ning Mao: Conception and design

Li Ding: Conception and design, Manuscript writing, Financial support, Final approval of manuscript

Heng Zhu: Conception and design, Data analysis and interpretation, Manuscript writing, Financial support, Final approval of manuscript

*Address correspondence to Heng Zhu, Beijing Institute of Radiation Medicine, Road Taiping

27, Beijing, 100850, P.R.China (email: zhudingdingabc@163.com);

** Address correspondence to Li Ding, Air Force Medical Center, PLA, Road Fucheng 30, Beijing, 100142, P.R.China (email: dingli7578@163.com).

\section{Funding}

This work was supported by National Natural Sciences Grants China (No. 81871771,

81572159,81500083 ,) and the Beijing Natural Sciences Foundation (No. 7182123,7192203). 


\begin{abstract}
The reconstruction of irradiated bone defects after settlement of skeletal tumors remains a significant challenge in clinical applications. In this study, we explored radiation-induced skeletal stem cell (SSC) stemness impairments and rescuing effects of ferulic acid (FA) on SSCs in vitro and in vivo. The immunophenotype, cell renewal, cell proliferation, and differentiation of SSCs in vitro after irradiation were investigated. Mechanistically, the changes in tissue regeneration-associated gene expression and MAPK pathway activation in irradiated SSCs were evaluated. The regenerative capacity of SSCs in the presence of FA in an irradiated bone defect mouse model was also investigated. We found that irradiation reduced CD140aand CD105-positive cells in skeletal tissues and mouse-derived SSCs. Additionally, irradiation suppressed cell proliferation, colony formation, and osteogenic differentiation of SSCs. The RNA-Seq results showed that tissue regeneration-associated gene expression decreased, and the western blotting results demonstrated the suppression of phosphorylated p38/MAPK and ERK/MAPK in irradiated SSCs. Notably, FA significantly rescued the radiation-induced impairment of SSCs by activating the p38/MAPK and ERK/MAPK pathways. Moreover, the results of imaging and pathological analyses demonstrated that FA enhanced the bone repair effects of SSCs in an irradiated bone defect mouse model substantially. Importantly, inhibition of the p38/MAPK and ERK/MAPK pathways in SSCs by specific chemical inhibitors partially abolished the promotive effect of FA on SSC-mediated bone regeneration. In summary, our findings reveal a novel function of FA in repairing irradiated bone defects by maintaining SSC stemness and suggest that the p38/MAPK and ERK/MAPK pathways contribute to SSCmediated tissue regeneration post-radiation.
\end{abstract}

Key words (5): Ferulic acid, skeletal stem cells, irradiation, bone defect, tissue repair

\title{
Significance Statement
}


Radiotherapy combined with surgery for the settlement of skeletal tumors usually leads to large bone defects and hampers wound healing. Skeletal stem cells (SSCs) have been defined as tissue-specific stem cells in skeletons and are highlighted in bone development and regeneration. Ferulic acid is a phytochemical with a wide range of therapeutic effects, including alleviation of radiation-induced oxidative stress and promotion of tissue regeneration. In the current study, promising data based on an in vitro cell model and an in vivo animal model demonstrates that ferulic acid alleviates radiation-induced impairment of SSCs and promotes SSC-mediated bone regeneration post-radiation. 


\section{Introduction}

The settlement of skeletal tumors often requires therapeutic surgical bone removal in association with radiation therapy ${ }^{1}$. Although the strategy proved effective in the treatment of skeletal tumors, it usually results in large bone defects and hampered wound healing because of extensive tissue cutoff and irradiation-induced tissue damage. To aesthetically and functionally reconstruct bone defects and repair surrounding tissues, a number of techniques have been developed, including autogenous bone graft transplantation and microanastomosed free-flap reconstitution ${ }^{2-5}$. However, surgical reconstruction brings a high risk of morbidity due to prolonged anesthesia and secondary trauma.

In recent years, mesenchymal stem cell (MSC)-based tissue repair has been used for bone regeneration post-radiation but has yielded controversial results. Zuo et al. reported that rat bone marrow mesenchymal stem cell (BMMSC)-derived exosomes were capable of alleviating radiation-induced bone loss by restoring the function of recipient BMMSCs $^{6}$. In another independent study, Liu et al. found that miR-34a promotes bone regeneration in irradiated bone defects by enhancing osteoblastic differentiation of BMMSCs in rats ${ }^{7}$. In contrast, Bléry et al. reported that the addition of a high concentration of BMMSCs did not improve bone regeneration in the irradiated areas of rats $^{8}$. These controversial reports may result from unstandardized protocols in different labs. Notably, the role of native tissue-specific stem cells in skeletons, as the pivotal population contributing to bone formation and regeneration, was overlooked.

Skeletal stem cells (SSCs) have been recently characterized as skeletal tissue-specific stem cells that play pivotal roles in skeletogenesis and osteochondrogenic repair ${ }^{9-16}$. Chan et al. identified a population of CD45-Ter119-Tie2-AlphaV + multipotent stem cells in murine bone and revealed the presence of SSC niches that regulate their activity ${ }^{10}$. A promising report from another lab demonstrated that Gremlin-1 defines a population of osteochondral reticular stem cells. Further functional studies suggest that these cells are closely involved in bone formation ${ }^{11}$. Furthermore, the identification of self-renewing and multipotent SSCs in human bones validates the presence of tissue-specific stem cells in skeletal tissue and highlights their 
contribution to skeletal regeneration ${ }^{16}$. We have pursued the identification of tissue-specific stem cells from skeletal tissues and explored their application in the past decade. Our previous studies revealed the pivotal role of SSCs in controlling the inflammatory response by targeting host immune cells ${ }^{17-23}$. Additionally, our recent work demonstrated that SSCs play a pivotal role in the regulation of bone remodeling by suppressing inflammatory osteoclastogenesis via the concerted action of cell adhesion molecules and osteoprotegerin ${ }^{24}$. However, there is little information available about the change in SSC stemness, including self-renewal and multidifferentiation, after irradiation and the related underlying regulatory factors thus far, which limits the current level of understanding regarding SSC-based bone regeneration.

Ferulic acid (FA) is a widely distributed hydroxycinnamic acid that exhibits potent antioxidant and therapeutic activities ${ }^{25}$. FA has been widely applied in the prevention of reactive oxygen species-related diseases, including cardiovascular diseases, diabetes mellitus, and cancers ${ }^{25}$. In addition, FA has alleviated radiation-induced stem cell damage. Ma et al. performed hematopoietic progenitor colony-forming cell assays to assess the reconstitution of murine bone marrow after radiation-induced myelosuppression. They found that FA treatment enhanced hematopoietic progenitor cell activity and promoted blood cell recovery in mice after irradiation by cobalt-60 gamma resources ${ }^{26}$. Moreover, FA promoted in vitro osteogenic differentiation of BMMSCs by inhibiting micro340 to induce $\beta$-catenin expression through hypoxia $^{27}$.

Given the fundamental role of SSCs in bone regeneration and the potential roles of FA in irradiation protection and osteogenic promotion, FA combined with SSCs may be an effective strategy for reconstructing irradiated bone defects. In the present study, we explore radiation-induced SSC impairments and the rescuing effects of FA on SSC-mediated bone regeneration by using an in vitro cell model and an in vivo animal model. Moreover, the cellular and molecular mechanisms underlying the protective effects of FA on SSCs were also investigated.

\section{Materials and Methods}




\section{Animals}

Normal inbred 8-week-old C57BL/6 mice $(\mathrm{n}=90)$ and 2-week-old C57BL/6 mice $(\mathrm{n}=16)$ were purchased from Beijing Vital River Laboratory Animal Technology Co., Ltd. All of the experiments were performed in accordance with the Academy of Military Medical Sciences Guide for Laboratory Animals.

\section{SSC preparation}

Murine SSCs were isolated from long bones according to our previously reported procedure $^{24}$. Briefly, femurs and tibiae from 2-week-old C57BL/6 mice were dissected, and the bone marrow cells were flushed out before the long bones were chopped and digested by collagenase II (Sigma, $0.1 \% \mathrm{vol} / \mathrm{vol}$ ) at $37^{\circ} \mathrm{C}$ for 1 hour. The released cells were discarded, and the digested bone chips were cultured in minimum essential medium eagle, alpha modification ( $\alpha$-MEM; Invitrogen, Carlsbad, CA) supplemented with 10\% fetal bovine serum (FBS) (HyClone, Logan, UT). The adherent cells from passages 3-6 were used for in vivo and in vitro experiments unless otherwise described. SSCs were also isolated from the femurs and tibiae of irradiated (cobalt-60) mice.

\section{FA preparation}

Ferulic acid (98\% purity, CAS 1135-24-6) was purchased from EFE BIO (Shanghai, China) and dissolved in DMSO to $20 \mathrm{mM}$ as a stock solution. The optimized concentrations of FA were screened by Cell Counting Kit 8 (CCK-8) assay with graded concentrations of FA (0, $10,20,30$ and $40 \mu \mathrm{M})$. It was determined that the optimal concentration of FA was $30 \mu \mathrm{M}$. This concentration was used in the experiments unless otherwise specified. In some experiments, the SSCs were pretreated with FA for 24 hours before usage.

\section{Immunophenotyping of SSCs}

To detect the effects of irradiation on the SSC immunophenotype in vivo, mice were 
irradiated with 2 Gy of gamma-radiation delivered at a rate of $0.98 \mathrm{~Gy} /$ minute. Femurs and tibiae were harvested 1 hour after irradiation for pathological analysis to determine the changes in CD140a-positive and CD105-positive cells in skeletal tissue. Additionally, SSCs were isolated from the long bones of irradiated mice (2 Gy) to investigate the SSC immunophenotype in vitro. For the mechanistic study, SSCs from normal mice were irradiated (2 Gy). Then, the SSCs were stained with PE- and PE-PerCP-conjugated monoclonal antibodies against mouse CD11b, CD31, CD44, CD45, CD80, CD105, CD140a and Sca-1 (eBio-Science, San Diego) according to the manufacturer's protocol. Events were collected by flow cytometry with a FACSCalibur system (Becton-Dickinson), and data analysis was conducted with FlowJo 10.0 software.

\section{CCK-8 assay}

The proliferation of SSCs was determined using the Cell Counting Kit 8 (CCK-8, Dojindo, Japan) according to the manufacturer's protocol. Briefly, a total of $2 \times 10^{3}$ irradiated SSCs or nonirradiated SSCs were seeded onto 96-well culture plates and added to the CCK-8 solution at a ratio of $100 \mu \mathrm{L}: 1 \mu \mathrm{L}$, and the plates were incubated at $37^{\circ} \mathrm{C}$ for 1 hour. Absorbance was then measured at a wavelength of $450 \mathrm{~nm}$ using a microplate reader. Five wells at each time point were assayed. The CCK-8 experiments were performed at different timepoints. FA was added at graded concentrations $(10,20,30$ and $40 \mu \mathrm{M})$ to investigate the potential effects of FA on SSC proliferation.

\section{Colony-forming unit fibroblast formation assay (CFU-F assay)}

In the current study, SSC self-renewal was assessed by a CFU-F assay. Aliquots 
$\left(2 \times 10^{2} /\right.$ well $)$ of irradiated SSC or nonirradiated SSC suspensions were added in triplicate into six-well culture plates and maintained in culture for 14 days. After crystal violet staining, visible colonies that were larger than $3 \mathrm{~mm}$ in diameter were counted using an inverted microscope. FA was added at graded concentrations to investigate the potential effects of FA on the self-renewal of SSCs.

\section{Multilineage differentiation experiments}

The osteogenic and adipogenic differentiation of irradiated SSCs and nonirradiated SSCs was determined by induction agents as previously described $(10,16)$. For osteogenic differentiation, SSCs were seeded into 48 -well plates at a cell density of $2 \times 10^{3} /$ well (five wells in each group) and incubated in osteogenic induction medium (10 mM glycerol-2-phosphate, $0.1 \mathrm{mM}$ dexamethasone, and $20 \mathrm{mM}$ ascorbic acid) for 14 or 28 days. For adipogenic differentiation, SSCs were seeded into 48 -well plates at a cell density of $1 \times 10^{4} /$ well (five wells in each group) and incubated in adipogenic induction medium for 14 days (1 mM isobutylmethylxanthine and $10-3 \mathrm{mM}$ dexamethasone). To evaluate osteogenesis at day 14 , the expression of the osteogenic marker alkaline phosphatase (ALP) was assessed using a histochemical kit (Sigma) per manufacturer's instructions. To evaluate adipogenesis at day 14, Oil Red O staining was performed according to previously described methods. To further determine the multiple-differentiation capacity of SSCs, the mRNA expression levels of the osteogenic genes Runx-2 and OCN and the adipogenic genes CEBP $/ \alpha$ and PPAR $\gamma$ were determined at day 10 using quantitative polymerase chain reaction (qPCR). FA was added at graded concentrations to investigate the potential effects of FA on the multidifferentiation of 
SSCs.

\section{RNA sequencing analyses of SSCs}

To further investigate the underlying mechanism of irradiation-mediated impairment of SSC properties, SSCs from irradiated or nonirradiated mice ( $2 \mathrm{~Gy}, 1$ hour after irradiation) were isolated, and high-throughput RNA sequencing analyses were performed. Next-generation sequencing library preparations and Illumina MiSeq sequencing were conducted at GENEWIZ, Inc. (Suzhou, China). DNA libraries were validated by an Agilent 2100 Bioanalyzer (Agilent Technologies, Palo Alto, CA, USA) and quantified with a Qubit 2.0 Fluorometer. DNA libraries were multiplexed and loaded onto an Illumina MiSeq instrument per manufacturer's instructions (Illumina, San Diego, CA, USA). Sequencing was performed using a $2 \times 300$ pairedend (PE) configuration; image analysis and base calling were conducted by MiSeq Control Software (MCS) embedded in the MiSeq instrument. All differentially abundant mRNAs were used for GO analysis to deepen the understanding of the molecular mechanism of cell biological information processes.

\section{SSC-microcryogel preparation}

To facilitate SSC transplantation, SSC microcryogels were prepared according to previous reports $^{28,29}$. Biodegradable gelatin microcryogels were obtained from Beijing Cytoniche Co., Ltd. (http://www.cytoniche.com/). Briefly, irradiated SSCs or FA-pretreated irradiated SSCs were harvested and resuspended at a concentration of $1 \times 10^{7}$ cells $/ \mathrm{ml}$. From each of these suspensions, $100-\mu \mathrm{L}$ was pipetted onto a dispersible and dissolvable porous microcarrier tablet (3D Table Trix, $20 \mathrm{mg} /$ tablet) to allow the suspension to be directly absorbed and hydrate the 
porous structures. The SSC microcryogels were then maintained in a humidified chamber and incubated at $37^{\circ} \mathrm{C}$ for 2 hours to allow for further cell attachment. After that, PBS was added to develop an injectable SSC microcryogel at a concentration of $20 \mathrm{mg}$ microcarrier/ml PBS.

\section{Transplantation of the SSC microcryogel in the irradiated bone defect murine model}

To explore the repairing capacity of SSC on irradiated bone defects, the femurs of mice were irradiated (2 Gy). Bone defect surgeries were conducted 1 hour after irradiation. A 1.5$\mathrm{mm}$ femoral defect was generated in both femurs. Nonirradiated mice that underwent surgery served as bone defect controls. A $50-\mu \mathrm{L}$ SSC microcryogel was injected into the local bone defect of irradiated mice unless otherwise described. The same concentration of microcryogel without SSCs was used as a control. To study the effects of FA on bone repair, FA solution was injected into irradiated bone defects. Mice were sacrificed at 1,2, and 3 weeks post-surgery, and the femurs were harvested for further micro-CT analysis and pathological evaluation.

\section{$\mu$ computerized tomography $(\mu \mathrm{CT})$ analysis}

The murine femurs were harvested and fixed overnight in 10\% neutral buffered formalin, washed twice in PBS, and kept in $70 \%$ ethanol at $4{ }^{\circ} \mathrm{C} . \mu \mathrm{CT}$ scanning and analysis were performed using a Scanco $\mu$ CT-40 (Scanco Medical). The femurs were scanned at a resolution of $8 \mu \mathrm{m}$, and reconstruction of three-dimensional (3D) images was performed using a standard convolution back-projection. The bone volume/tissue volume ratio (BV/TV) was calculated by measuring 3D distances directly in the trabecular network.

\section{Histological Examination and Immunohistochemistry}


All femurs were fixed in 4\% paraformaldehyde for 3 days, decalcified in 10\% EDTA solution for 7 days, and then embedded in paraffin. The femurs were sectioned into 6-mm slices that were subjected to $\mathrm{HE}$ and Masson staining. In addition, osteogenic regeneration and SSC involvement were further evaluated by immunohistochemical analyses of Col-I and OCN.

\section{Real-Time Quantitative Polymerase Chain Reaction}

qPCR was performed to further investigate SSC differentiation and its underlying mechanisms. The procedure was performed according to previous studies. Briefly, total RNA was extracted using TRIzol reagent (Fermentas) and reverse transcribed using the mRNA Selective PCR Kit (TaKaRa) according to the manufacturer's instructions. Murine Runx2, OCN, CEBP/ $\alpha$, PPAR $\gamma$, Fgf2, Stc1, Bmpr1b, Clec3b, Lama5, Gata2, Nanog, and Sox2 cDNA were amplified by real-time qPCR using a SYBR PCR Master Mix Kit (Sigma-Aldrich) and a 7500 Real-Time PCR Detection System (Applied Biosystems, ABI). The primers were synthesized by Invitrogen and are shown in supplementary Table 1. The mRNA levels were normalized to the value of glyceraldehyde-3-phosphate dehydrogenase.

\section{Western-blotting}

Normal mouse-derived SSCs were seeded into cell culture plates $\left(5 \times 10^{5}\right.$ cells/well) and irradiated with 2 Gy Co 60. FA was added to the SSC culture at the earliest possible time after irradiation. Nonirradiated SSCs were used as control. The SSCs were collected at 60 min postirradiation. Protein lysis buffer (BioRad, Hercules, CA) was added, and the thawed lysates were vortexed and centrifuged. Proteins were separated by $10 \%$ sodium dodecyl sulfatepolyacrylamide gel electrophoresis (SDS-PAGE) and transferred onto nitrocellulose 
membranes. The membranes were blocked by incubation with $5 \% \mathrm{wt} / \mathrm{vol}$ nonfat dry milk. The membranes were then incubated with anti-phospho-JNK (P-JNK), anti-JNK, anti-phospho-p38 (P-p38), anti-p38, anti-phospho-ERK (P-ERK), anti-ERK, and anti-GAPDH antibodies (Cell Signaling Technology) at the appropriate dilutions overnight at $4{ }^{\circ} \mathrm{C}$. After incubation, the membranes were washed in Tris buffered saline with Tween-20 (TBST). Horseradish peroxidase-conjugated secondary antibodies were added to the membranes in $5 \%$ nonfat dry milk in TBST.

\section{Statistical analysis}

Data are represented as the mean values with standard deviations. Statistical significance was analyzed using Student's t-test. P values less than 0.05 were considered significant.

\section{Results}

\section{Irradiation-induced stemness impairment of skeletal stem cells}

CD105 and CD140a are pivotal cell surface markers to identify tissue specific stem/progenitor cells in skeletons as previously reported ${ }^{18,19,23,24}$. As shown in Figure 1A and 1B, 2 Gy irradiation caused a significant reduction in the number of in situ $\mathrm{CD} 105^{+}$and $\mathrm{CD} 140 \mathrm{a}^{+}$cells by $>30 \%$ in murine femurs $\left({ }^{*} p<0.05, * * p<0.01, * * * p<0.001\right)$. Additionally, the results of immunohistochemical staining of bone slices demonstrated that irradiation led to a decrease in the number of $\mathrm{CD} 105^{+}$and $\mathrm{CD} 140 \mathrm{a}^{+}$cells in murine femurs in a radiation dosedependent manner (Figure 1A and 1B). To minimize secondary effects in vivo, murine SSCs were isolated 1 hour post-irradiation. The immunophenotype, colony formation, cell proliferation, and osteo-adipogenic differentiation were evaluated. The results of 
immunophenotyping showed that irradiation (2 Gy) remarkably decreased the ratios of CD105 and CD140a cells in SSCs (Figure 1C) $(* * p<0.01)$. In addition, the CFU-F assay showed that irradiated SSCs exhibited decreased colony formation capacity relative to that of nonirradiated SSCs (Figure 1D and 1E) $(* p<0.05, * * p<0.01, * * * p<0.001)$. Consistent with the results of CFU-F experiments, the expression of self-renewal-related genes, including Nanog and Sox2, remarkably decreased after irradiation (Figure $1 \mathrm{~F})(* * p<0.01, * * * p<0.001)$. Furthermore, the CCK-8 data demonstrated that irradiation inhibited cell proliferation (Figure 1G) ${ }^{* *} p<$ 0.01, *** $p<0.001$ ). Moreover, irradiation inhibited ALP activity (Figure 1H) and the expression of osteogenic genes, including Runx-2 and OPN (Figure 1I), in SSCs. The suppressive effect was dose-dependent upon irradiation (Figure $1 \mathrm{H}$ and $1 \mathrm{I})\left({ }^{*} p<0.05,{ }^{* *} p<\right.$ $0.01, * * * p<0.001)$. No obvious influences of irradiation (2Gy)on adipogenic differentiation of SSC were observed (Figure S1A and S1B). Therefore, these results suggest that irradiation caused significant impairment of SSC stemness.

\section{Differential gene expression and activation of MAPK pathways characterize the response}

\section{of skeletal stem cells to irradiation}

To explore the underlying mechanisms by which irradiation impaired SSC properties, the mRNA expression profile of SSCs after irradiation was identified by transcriptome sequencing. A total of 55,574 genes were examined in the SSC $(n=3)$ group and irradiated SSC $(2 \mathrm{~Gy}, \mathrm{n}=$ 3) group. The false discovery ratio was less than 0.05 . The difference multiple was greater than 2-fold change to define significantly different proteins. A total of 638 genes were upregulated and 198 genes were downregulated in the irradiated SSC group compared with the SSC control 
group. The data in Figure S2A and S2B present the differentially expressed genes in heatmap and volcano plot format between SSC and irradiated SSC. Notably, the expression of osteogenesis-related genes, including Fgf2, Stc1, Bmpr1b, and Clec3b, and the cell proliferation-related genes Lama5 and Gata2 were significantly downregulated (Figure 2A). The results of Gene Ontology (GO) analysis showed that all of the differentially abundant genes between the SSCs and the irradiated SSCs were mainly involved in ossification, regulation of ossification, osteoblast differentiation, biomineral tissue development, and bone mineralization, etc (Figure 2B). The results of qPCR validated the gene expression of RNA sequencing (Figure $2 \mathrm{C})(* * p<0.01, * * * p<0.001)$.

Further KEGG analysis suggested that the MAPK signaling pathway, TGF- $\beta$ signaling pathway and cytokine-cytokine receptor signaling pathway were closely involved in the irradiation-induced changes in SSC properties (Figure 2D).

Based on the KEGG analysis results and the important roles of MAPK signaling in bone formation and response to stress, the phosphorylation of the p38/MAPK, ERK/MAPK, and JNK/SAPK pathways was investigated. The results showed that irradiation (2 Gy) significantly inhibited the phosphorylation of p38/MAPK and ERK/MAPK in SSCs. In addition, there was obvious suppression of both the phosphorylated JNK and total JNK proteins in irradiated SSCs

(Figure 2E) $(* * * p<0.001)$.

\section{Ferulic acid (FA) partially alleviates irradiation-induced stemness impairment of}

\section{skeletal stem cells}

Because FA has been proven to be closely involved in radiation protection and bone formation in previous studies ${ }^{26,27}$, we investigated its role in the regulation of SSC stemness in the current 
study. As shown in Figure 3A, FA promoted SSC proliferation in an FA dose-dependent manner $\left(* p<0.05,{ }^{* *} p<0.01, * * * p<0.001\right)$. Notably, the CCK-8 results demonstrated that FA significantly rescued the irradiation-induced proliferation inhibition of SSCs (Figure 3A) $\left({ }^{*} p<0.05,{ }^{* *} p<0.01,{ }^{* *} p<0.001\right)$. Furthermore, ALP activity assays showed that FA (30 $\mu \mathrm{M})$ promoted osteogenic differentiation of SSCs and irradiated SSCs (Figure 3B). The realtime PCR results demonstrated that FA enhanced the expression of osteogenic Runx-2 and OPN in SSCs (Figure 3C) $(* * p<0.01, * * * p<0.001)$. The colony formation capacity partially reflects SSC stemness. We found that in the presence of FA, SSCs developed more CFU-F than their counterparts in the absence of FA (Figure 3D) $\left({ }^{* * *} p<0.001\right)$. Importantly, FA addition partially restored the colony formation that was compromised by irradiation, indicating that FA alleviates irradiation-induced SSC stemness impairment (Figure 3D). Moreover, FA regulated the expression of the self-renewal-related genes Nanog and Sox2 in SSCs and irradiated SSCs (Figure 3E) $\left({ }^{*} p<0.05, * * p<0.01, * * * p<0.001\right)$.

FA partially rescues irradiation-induced stemness impairment of SSCs by activating the p38/MAPK and ERK/MAPK pathways

To explore the association of the MAPK pathways and the promising FA-mediated effects on SSC, FAs were added to the SSC culture system. As shown in Figure 4A, supplementation with FA $(30 \mu \mathrm{M})$ significantly restored the phosphorylation of p38/MAPK and ERK/MAPK in irradiated SSCs (Figure 4A) $\left({ }^{*} p<0.05, * * * p<0.001\right)$. However, no obvious effects of FA on the phosphorylated JNK and total JNK proteins in nonirradiated SSCs and irradiated SSCs were observed.

To further clarify the innate relations of FA-induced activation of MAPK signaling and SSC 
biological properties, specific chemical inhibitors, including SB203580 (for p38/MAPK) and PD98059 (for ERK/MAPK), were added to the SSC culture system. The expression of genes that control SSC proliferation and differentiation, cell proliferation, osteogenic differentiation, and the colony formation capacity of SSCs were evaluated. The real-time results in Figure 4B show that inhibition of p38/MAPK and ERK/MAPK signaling by specific inhibitors abolished the promoting effects of FA on the mRNA expression of Stc1 and Bmpr1b, indicating that these genes may be involved in FA-mediated regulation of SSC osteogenic differentiation and cell proliferation via MAPK signaling $(* p<0.05, * * p<0.01, * * * p<0.001)$. Additionally, the CCK-8 assay data demonstrated that SB203580 and PD98059 blocked the rescued effects on cell proliferation in irradiated SSCs substantially (Figure 4C) $(* * p<0.01, * * * p<0.001)$. Moreover, SB203580 and PD98059 significantly suppressed ALP activity and the mRNA expression of Run2 and OPN in irradiated cells in the presence of FA (Figure 4D, 4E and 4F) $\left({ }^{* *} p<0.01,{ }^{* * *} p<0.001\right)$. Further, the colony numbers of irradiated SSCs in the FA-buffered culture system dramatically decreased in the presence of SB203580 and PD98059 (Figure 4G and $4 \mathrm{H})\left({ }^{* * *} p<0.001\right)$. Consistent with the change in colony formation assays, the mRNA expression of Nanog and Sox 2 was also downregulated with the addition of specific inhibitors of the p38/MAPK and ERK/MAPK pathways, respectively (Figure 4I and 4J) $\left({ }^{* *} p<0.01\right.$, *** $p<0.001)$. Thus, these data suggest that FA enhanced osteogenic differentiation and proliferation partially via activation of the p38/MAPK and ERK/MAPK pathways.

\section{FA primed SSCs combined with microcryogel promotes bone repair in a murine irradiated bone defect model}

Although in vitro studies have shown that FA is capable of maintaining SSC stemness, it 
remains unknown whether this is the case in vivo. Therefore, the effects of FA on SSC-mediated bone regeneration in an irradiated bone defect animal model were explored. FA-treated irradiated SSCs or irradiated SSCs were combined with a microniche to develop an injectable SSC-microcryogel (Figure 5A).

Bone regeneration in the defect was evaluated by micro-CT at 1, 2, and 3 weeks after SSC microcryogel transplantation. As shown in Figure 5B, more regenerated bone was observed in the irradiated-SSC-combined-with-microcryogel group than in the negative control group and the microcryogel group. Promisingly, transplantation of FA-treated irradiated SSCs combined with microcryogel yielded strengthened bone formation in the defect compared with its counterpart without FA treatment (Figure 5B). Additionally, BV/TV was higher in the irradiated SSC-microcryogel group than in the negative control group and microcryogel group. Similarly, the FA-treated irradiated SSC microcryogel group showed a greater increase in $\mathrm{BV} / \mathrm{TV}$ than the irradiated SSC-microcryogel group (Figure 5C) $(* * p<0.01, * * * p<0.001)$. To further explore bone regeneration in the defect, HE and Masson staining as well as immunohistochemical analyses of Col-I and OCN were performed. The histological data showed that only fibrous-like connective tissues filled the defect even at week 3 engrafted with microcryogel. Small bone nodules were observed in the bone defect transplanted with the irradiated SSC microcryogel (Figure 6A). Notably, bone-like tissues filled most of the defects engrafted with FA-treated irradiated SSC microcryogels. In addition, the results of Masson's trichrome and Col-I staining showed that FA promoted the formation of collagen fibers in new bones, which contributed to bone regeneration in defects (Figure 6B). The quantitative analysis of new bone was further performed according to the results of HE and Masson's trichrome 
staining. The femur defect repair percentage was calculated by Image-Pro Plus. Figure 6C shows that new bone formation in the defect engrafted by FA-treated irradiated SSC microcryogels $(28.3 \pm 1.1 \%$ at day $7,32.6 \pm 1.9 \%$ at day $14,38.6 \pm 2.1 \%$ at day 21$)$ was higher than in defect grafts engrafted by irradiated SSC microcryogels $(21.6 \pm 2.1 \%$ at day $7,24.0 \pm 2.9 \%$ at day $14,28.9 \pm 3.2 \%$ at day 21$) \quad(*, P<0.05, * *, P<0.01, * * *, P<0.001)$

\section{FA partially rescued the bone regenerative capacity of irradiated SSCs in vivo via the p38/MAPK and ERK/MAPK pathways}

To explore the underlying mechanisms that control FA-SSC-mediated bone regeneration in vivo, specific chemical inhibitors of p38/MAPK (SB203580) and ERK/MAPK (PD98059) were added to the FA-buffered irradiated SSC culture system for 12 hours before preparing FAtreated irradiated SSC microcryogels. The bone regeneration of SSCs in each group was then evaluated. The imaging results showed that inactivation of p38/MAPK and ERK/MAPK impaired FA/SSC-mediated structural reconstitution in bone defects (Figure S3A, S3B, and S3C). Further pathological data demonstrated that SB203580 and PD98059 partially abolished the promotive effects of FA on the bone regenerative cap of irradiated SSCs in vivo (Figure S3A, S3B, and S3C). The data of newborn bones showed that blockage of p38/MAPK and ERK/MAPK pathways by specific inhibitors in FA treated irradiated-SSC significant abolished the bone regenerative activities in bone defects $(33.5 \pm 3.6 \%$ vs $19.8 \pm 1.1 \%$ of SB203580, vs $22.1 \pm 0.9 \%$ of PD98059) (Figure S3D) $(* * *, P<0.001)$.

\section{Discussion}

In the current study, we found that irradiation injured SSC stemness by targeting MAPK 
signaling in vitro and in vivo. In addition, FA was capable of maintaining SSC stemness after irradiation. Promisingly, our findings showed that FA restored the bone regenerative capacity of SSCs by activating MAPK pathways.

SSCs have been recently characterized as innate stem cells residing in skeletons that contribute to bone development, bone fracture repair, and bone remodeling ${ }^{9-16,24}$. However, the response of SSCs to irradiation, the role of SSCs in the settlement of radiation-induced bone injury, and the underlying mechanisms have remained largely unknown. Green et al. found that irradiation devastated the adult stem cell pools in mice preceding the collapse of trabecular bone quality and quantity ${ }^{30}$. Although the impact of irradiation on hematopoietic stem cells was observed, few phenotypes of skeletal tissue-derived stem cells were reported in their study. Additionally, Marecic et al. harvested dissociated progenitor cells, fracture-induced bone, cartilage, and stromal progenitor (f-BCSP) from complete femurs by collagenase digestion after bone fracture and irradiation ${ }^{31}$. They demonstrated that f-BCSP expansion was reduced significantly in irradiated versus nonirradiated calluses in a murine bone fracture model. Both callus development and BCSP expansion remained impaired 3 months post-irradiation ${ }^{31}$. Furthermore, Chandra et al. labeled all mesenchymal lineage cells in the endosteal bone marrow using a lineage tracing approach and found that radiation shifted the differentiation fate of mesenchymal progenitors to adipocytes and arrested their proliferation ability ${ }^{32}$. However, the stem/progenitor cells described in these reports are mainly from bone morrow or mixed with bone marrow stem cells. In our study, the bone marrow cells were removed by cavity flushing and collagenase digestion. Only SSCs, innate stem cells in bone tissues, were isolated and used in the subsequent experiments. Notably, our data showed that SSC is very sensitive to 
irradiation and exhibits impaired stemness, which adds novel information to understanding bone osteoporosis and hindered bone repair post-irradiation. Most importantly, the data of cellular and animal experiments suggest that SSCs may be a potential target for the settlement of irradiated bone defects.

Stem cells have been proven to be one of the important targets of drug-based therapy. Mukherjee et al. targeted the stem/progenitor population by using bortezomib, a clinically available proteasome inhibitor used in the treatment of multiple myeloma ${ }^{33}$. They found that bortezomib induced MSCs to preferentially undergo osteoblastic differentiation in vitro, and mice implanted with MSCs showed increased ectopic ossicle and bone formation when recipients received low doses of bortezomib ${ }^{33}$. Another study demonstrated that transplantation of pioglitazone-pretreated MSCs significantly enhanced cardiac transdifferentiation in vitro and improved cardiac function in a myocardial infarction model in vivo ${ }^{34}$. Additionally, treatment of MSCs with angiotensin receptor blockers has been proven to significantly improve the efficiency of cardiomyogenic transdifferentiation and cardiac function via angiogenesis ${ }^{35}$. Based on our understanding of SSC biology and previous research findings, we targeted irradiated SSCs in vitro by FA and subsequently evaluated their contribution to bone regeneration in vivo. First, FA was used in the current study because of its biodirectional effects of radiation protection and osteogenic promotion ${ }^{26,27}$. In addition, the sodium salt of FA, sodium ferulate, is a compound widely used in traditional Chinese medicine and has been approved by the National Medical Products Administration (NMPA) for clinical applications ${ }^{25}$. Notably, mechanistic studies have demonstrated a versatile genetic control system in mammalian cells and mice that is responsive to clinically licensed sodium ferulation ${ }^{25}$. Secondly, in the current 
study, mirocryogels were used to build stem cell-scaffold constructs that were transplanted into bone defects because the matrix is indispensable for tissue regeneration. Microcryogels have been successfully used in previous studies by virtue of their properties of cell protection, cell retention and survival, which ultimately improves the therapeutic functions of stem cells $\mathrm{s}^{28,29}$. Thirdly, the irradiated bone defects were repaired using FA-primed allogenic SSCs and scaffolds, instead of intravenously administered FA, to recruit host SSCs because the limited endogenous SSC number in the local site may limit the efficiency of bone repair. However, FA infusion may be suitable for alleviating total body radiation-induced SSC injury and subsequent osteoporosis.

In addition to the results of cellular and animal experiments, further mechanistic data revealed the signaling pathways by which FA controls the regenerative effects of SSC. Accumulated evidence has proven that the p38 MAPK pathway is required for normal skeletogenesis in mice. Greenblatt et al. reported that mice with a deletion of the MAPK pathway members displayed remarkably reduced bone mass due to defective osteogenic differentiation. These findings suggest that p38/MAPK signaling is essential for bone formation in vivo ${ }^{36}$. In addition, ERK and p38 MAPKs cause phosphorylation of RUNX2, which promotes osteoblast differentiation. ERK also activates RSK2, which in turn phosphorylates ATF4, to enhance late-stage osteoblast synthetic functions ${ }^{37}$. In addition to normal skeletogenesis, MAPK signaling plays a pivotal role in bone repair. Acting as signal transductors from multiple growth factors or adhesion molecules, the MAPK pathway plays crucial functions during bone healing post-fracture ${ }^{38}$. Moreover, our previous work showed that inhibition of the p38/MAPK pathway caused serious osteogenic suppression ${ }^{39}$. Remarkably, in the present study, FA 
reversed radiation-induced MAPK suppression and SSC stemness injury. Further inhibition of the MAPK pathway by a specific chemical inhibitor significantly abolished the rescuing effect of FA on irradiated SSCs. Our data are consistent with previous reports that MAPK signaling plays an important role in bone regeneration and reveals the novel role of MAPK in FAmediated anti-radiation and bone repair.

Nevertheless, we must acknowledge that there are several limitations in our study. First, although we have identified that MAPK pathways mainly contribute to the regulatory effects of FA on SSC, the potential role of other pathways, such as the TGF- $\beta$ /SMAD and NF- $\mathrm{kB}$ pathways, should be investigated in future studies. Secondly, the contribution of endogenous SSCs and other stem cells in the host needs to be clarified, and more animal models, including genetically modified mice, may be helpful in addressing this issue. Thirdly, to explore the translational value of our findings, more drugs should be introduced into future studies, and their effects on SSCs need to be compared with FA.

\section{Summary}

In the present study, we report a new role for FA in maintaining SSC stemness after irradiation. In addition, our study reveals the underlying cellular and molecular mechanisms by which FA controls bone reconstitution post-irradiation by SSCs. Our data suggest that targeting SSCs may be a novel strategy in treating irradiated bone injury.

\section{Acknowledgments}

This study was supported by the National Natural Sciences Grants China (No. 81871771, 81572159,81500083,) and the Beijing Natural Sciences Foundation (No. 7182123,7192203). 


\section{Disclosure of Potential Conflicts of Interest}

The authors declare no competing financial interests.

\section{Data Availability Statement}

The data that support the findings of this study are available from the corresponding author upon reasonable request.

\section{References}

1. Mottard S, Grimer RJ, Abudu A, et al. Biological reconstruction after excision, irradiation and reimplantation of diaphyseal tibial tumours using an ipsilateral vascularised fibular graft. J Bone Joint Surg Br. 2012;94(9):1282-1287.

2. R Schuh, J Panotopoulos, S E Puchner, et al. Vascularised or non-vascularised autologous fibular grafting for the reconstruction of a diaphyseal bone defect after resection of a musculoskeletal tumour. Bone Joint J . 2014;96-B(9):1258-1263.

3. Kotb SZ, Mostafa MF. Recycling of extracorporeally irradiated autograft for malignant bone tumors: long-term follow-up. Ann Plast Surg. 2013;71(5):493-499.

4. Wu PK, Chen $\mathrm{CF}$, Chen $\mathrm{CM}$, et al. Intraoperative Extracorporeal Irradiation and Frozen Treatment on Tumor-bearing Autografts Show Equivalent Outcomes for Biologic Reconstruction. Clin Orthop Relat Res. 2018;476(4):877-889.

5. Goodwin ML, Gundavda MK, Reddy R, et al. Extracorporeal radiation and reimplantation: a safe and viable option for reconstruction after sacral tumor resection? Ann Transl Med. 2019;7(10):229. 
6. Zuo R, Liu M, Wang Y, et al. BM-MSC-derived exosomes alleviate radiation-induced bone loss by restoring the function of recipient BM-MSCs and activating Wnt/beta-catenin signaling. Stem Cell Res Ther. 2019;10(1):30.

7. Liu H, Dong Y, Feng X, et al. miR-34a promotes bone regeneration in irradiated bone defects by enhancing osteoblastic differentiation of mesenchymal stromal cells in rats. Stem Cell Res Ther. 2019;10(1):180.

8. Bléry P, Corre P, Malard O, et al. Evaluation of new bone formation in irradiated areas using association of mesenchymal stem cells and total fresh bone marrow mixed with calcium phosphate scaffold. J Mater Sci Mater Med. 2014;25(12):2711-2720.

9. Bianco P, Robey PG. Skeletal stem cells. Development 2015;142:1023-1027.

10. Chan CK, Seo EY, Chen JY, et al. Identification and specification of the mouse skeletal stem cell. Cell 2015;160:285-298.

11. Worthley DL, Churchill M, Compton JT, et al. Gremlin 1 identifies a skeletal stem cell with bone, cartilage, and reticular stromal potential. Cell. 2015;160:269-284.

12. Tang Y, Feinberg T, Keller ET, et al. Snail/Slug binding interactions with YAP/TAZ control skeletal stem cell self-renewal and differentiation. Nat Cell Biol. 2016;18:917-929.

13. Maruyama T, Jeong J, Sheu TJ, et al. Stem cells of the suture mesenchyme in craniofacial bone development, repair and regeneration. Nat Commun. 2016;7:10526.

14. Shi Y, He G, Lee WC, et al. Gli 1 identifies osteogenic progenitors for bone formation and fracture repair. Nat Commun. 2017;8:2043.

15. Debnath S, Yallowitz AR, McCormick J, et al. Discovery of a periosteal stem cell mediating intramembranous bone formation. Nature 2018;562:133-139. 
16. Chan CKF, Gulati GS, Sinha R, et al. Identification of the Human Skeletal Stem Cell. Cell 2018;175:43-56.e21.

17. Guo Z, Li H, Li X, et al. In vitro characteristics and in vivo immunosuppressive activity of compact bone-derived murine mesenchymal progenitor cells. Stem Cells 2006;24:992-1000

18. Zhu H, Guo ZK, Jiang XX, et al. A protocol for isolation and culture of mesenchymal stem cells from mouse compact bone. Nat Protoc. 2010;5:550-560.

19. Wang K, Li J, Li Z, et al. Chondrogenic Progenitor Cells Exhibit Superiority Over Mesenchymal Stem Cells and Chondrocytes in Platelet-Rich Plasma Scaffold-Based Cartilage Regeneration. Am J Sports Med. 2019:363546519854219.

20. Li H, Guo Z, Jiang X, et al. Mesenchymal stem cells alter migratory property of $\mathrm{T}$ and dendritic cells to delay the development of murine lethal acute graft-versus-host disease. Stem Cells. 2008;26:2531-2541.

21. Li H, Jiang Y, Jiang X, et al. CCR7 guides migration of mesenchymal stem cell to secondary lymphoid organs: a novel approach to separate GvHD from GvL effect. Stem Cells. $2014 ; 32(7): 1890-1903$.

22. Zhu H, Yang F, Tang B, et al. Mesenchymal stem cells attenuated PLGA-induced inflammatory responses by inhibiting host DC maturation and function. Biomaterials 2015;53:688-698.

23. Wang YX, Zhao ZD, Wang Q, et al. Biological potential alterations of migratory chondrogenic progenitor cells during knee osteoarthritic progression. Arthritis Res Ther. 2020;22(1):62.

24. Li X, Ding L, Wang YX, et al. Skeletal stem cell-mediated suppression on inflammatory 
osteoclastogenesis occurs via concerted action of cell adhesion molecules and osteoprotegerin. Stem Cells Transl Med. 2020;9(2):261-272.

25. Wang Y, Liao S, Guan N, et al. A versatile genetic control system in mammalian cells and mice responsive to clinically licensed sodium ferulate. Sci Adv. 2020;6(32):eabb9484.

26. Ma Z, Hong Q, Wang Y, et al. Effects of ferulic acid on hematopoietic cell recovery in whole-body gamma irradiated mice. Int J Radiat Biol. 2011;87(5):499-505.

27. Du K, Li Z, Fang X, et al. Ferulic acid promotes osteogenesis of bone marrow-derived mesenchymal stem cells by inhibiting microRNA-340 to induce $\beta$-catenin expression through hypoxia. Eur J Cell Biol. 2017;96(6):496-503.

28. Li Y, Liu W, Liu F, et al. Primed 3D injectable microniches enabling low-dosage cell therapy for critical limb ischemia. Proc Natl Acad Sci U S A. 2014;111(37):13511-13516.

29. Zeng Y, Chen C, Liu W, et al. Injectable microcryogels reinforced alginate encapsulation of mesenchymal stromal cells for leak-proof delivery and alleviation of canine disc degeneration. Biomaterials. 2015;59:53-65.

30. Green DE, Adler BJ, Chan ME, et al. Devastation of adult stem cell pools by irradiation precedes collapse of trabecular bone quality and quantity. J Bone Miner Res. 2012;27(4):749-759.

31. Marecic O, Tevlin R, McArdle A, et al. Identification and characterization of an injuryinduced skeletal progenitor. Proc Natl Acad Sci U S A. 2015;112(32):9920-9925.

32. Chandra A, Lin T, Young T, et al. Suppression of Sclerostin Alleviates Radiation-Induced Bone Loss by Protecting Bone-Forming Cells and Their Progenitors Through Distinct Mechanisms. J Bone Miner Res. 2017;32(2):360-372. 
33. Mukherjee S, Raje N, Schoonmaker JA,et al. Pharmacologic targeting of stem/progenitor population in vivo is associated with enhanced bone regeneraiton in mice. J Clin Invest. 2008;118(2):491-504.

34. Shinmura D, Togashi I, Miyoshi S, et al. Pretreatment of human mesenchymal stem cells with pioglitazone improved efficiency of cardiomyogenic transdifferentiation and cardiac function. Stem Cells. 2011 Feb;29(2):357-366.

35. Numasawa Y, Kimura T, Miyoshi S, et al. Treatment of human mesenchymal stem cells with angiotensin receptor blocker improved efficiency of cardiomyogenic transdifferentiation and improved cardiac function via angiogenesis. Stem Cells. $2011 ; 29(9): 1405-1414$.

36. Greenblatt MB, Shim JH, Zou W, et al.The p38 MAPK pathway is essential for skeletogenesis and bone homeostasis in mice. J Clin Invest. 2010;120(7):2457-2473.

37. Greenblatt MB, Shim JH, Glimcher LH. Mitogen-activated protein kinase pathways in osteoblasts. Annu Rev Cell Dev Biol. 2013;29:63-79.

38. Rodríguez-Carballo E, Gámez B, Ventura F. p38 MAPK Signaling in Osteoblast Differentiation Front Cell Dev Biol. 2016;4:40.

39. $\mathrm{Xu} \mathrm{F}, \mathrm{Zhu} \mathrm{H}, \mathrm{Li} \mathrm{X}$, et al. Intercellular adhesion molecule-1 inhibits osteogenic differentiation of mesenchymal stem cells and impairs bio-scaffold-mediated bone regeneration in vivo. Tissue Eng Part A. 2014;20(19-20):2768-2782.

Figure 1 Irradiation caused stemness impairment of skeletal stem cells 
Irradiation caused a significant reduction in the number of in situ $\mathrm{CD} 105^{+}$and $\mathrm{CD} 140 \mathrm{a}^{+}$cells in murine femurs at a radiation dose-dependent manner (Figure 1A and 1B). Additionally, irradiation remarkably decreased the ratios of CD105 and CD140a cells in SSCs in vitro (Figure 1C). Furthermore, the CFU-F assay showed that irradiated SSCs exhibited decreased colony formation capacity relative to that of nonirradiated SSCs (Figure 1D and 1E). Moreover, the expression of self-renewal-related genes, Nanog and Sox2, remarkably decreased after irradiation (Figure 1F). The CCK-8 data demonstrated that irradiation inhibited cell proliferation (Figure 1G). Further data showed that irradiation inhibited ALP activity (Figure $1 \mathrm{H})$ and the expression of osteogenic genes, including Runx-2 and OPN (Figure 1I), in SSCs at dose-dependent manner upon irradiation (Figure 1H and 1I). Bars in Figure 1A, 1D and 1H represent $200 \mu \mathrm{m}, 500 \mu \mathrm{m}$ and $200 \mu$, respectively. ${ }^{*} p<0.05,{ }^{* *} p<0.01,{ }^{* * *} p<0.001$.

\section{Figure 2 Differential gene expression and activation of MAPK pathways characterize the}

\section{response of skeletal stem cells to irradiation}

The results of mRNA sequencing ( $2 \mathrm{~Gy}, \mathrm{n}=3$ ) showed that the expression of osteogenesisrelated genes, including Fgf2, Stc1, Bmpr1b, and Clec3b, and the cell proliferation-related genes Lama5 and Gata2 were significantly downregulated (Figure 2A). The results of Gene Ontology (GO) analysis showed that all of the differentially abundant genes between the SSCs and the irradiated SSCs were mainly involved in ossification, regulation of ossification, osteoblast differentiation, biomineral tissue development, and bone mineralization, etc (Figure 2B). The data of qPCR validated the gene expression of RNA sequencing (Figure 2C). Further KEGG analysis suggested that the MAPK signaling pathway, TGF- $\beta$ signaling pathway and 
cytokine-cytokine receptor signaling pathway were closely involved in the irradiation-induced changes in SSC properties (Figure 2D). The western-blotting data showed that irradiation (2 Gy) significantly inhibited the phosphorylation of p38/MAPK and ERK/MAPK in SSCs. There was obvious suppression of both the phosphorylated JNK and total JNK proteins in irradiated SSCs (Figure 2E) $* * p<0.01, * * * p<0.001$.

Figure 3 Ferulic acid (FA) partially alleviates irradiation-induced stemness impairment of skeletal stem cells

FA promoted SSC proliferation in an FA dose-dependent manner (Figure 3A). Notably, the CCK-8 results showed that FA significantly rescued the irradiation-induced proliferation inhibition of SSCs (Figure 3A). Furthermore, the data of ALP staining showed that FA (30 $\mu \mathrm{M})$ promoted osteogenic differentiation of SSCs and irradiated SSCs (Figure 3B). The qPCR results demonstrated that FA enhanced the expression of osteogenic Runx-2 and OPN in SSCs (Figure 3C). Moreover, SSCs developed more CFU-F in the presence of FA (Figure 3D). Importantly, FA supplementation partially restored the colony formation that was compromised by irradiation (Figure 3D). Moreover, FA regulated the expression of the self-renewal-related genes Nanog and Sox2 in SSCs and irradiated SSCs (Figure 3E). Bars in Figure 3B and 3D represent $200 \mu \mathrm{m}$ and $500 \mu \mathrm{m}$, respectively. ${ }^{*} p<0.05, * * p<0.01, * * * p<0.001$.

Figure 4 FA partially rescues irradiation-induced stemness impairment of SSCs by activating the p38/MAPK and ERK/MAPK pathways

Supplementation with FA $(30 \mu \mathrm{M})$ significantly restored the phosphorylation of p38/MAPK and ERK/MAPK in irradiated SSCs (Figure 4A). No obvious effects of FA on the 
phosphorylated JNK and total JNK proteins in nonirradiated SSCs and irradiated SSCs were observed. Inhibition of p38/MAPK and ERK/MAPK signaling by specific inhibitors (SB203580 and PD98059) abolished the promoting effects of FA on the mRNA expression of Stc1 and Bmpr1b. Additionally, the CCK-8 assay data demonstrated that SB203580 and PD98059 blocked the rescued effects on cell proliferation in irradiated SSCs (Figure 4C). Moreover, SB203580 and PD98059 significantly suppressed ALP activity and the mRNA expression of Run2 and OPN in irradiated cells in the presence of FA (Figure 4D, 4E and 4F). Further, the colony numbers of irradiated SSCs in the FA-buffered culture system, and the mRNA expression of Nanog and Sox2 dramatically decreased in the presence of SB203580 and PD98059, respectively (Figure 4G, 4H, 4I and 4J). Bars in Figure 4D and 4G represent $200 \mu \mathrm{m}$ and $500 \mu \mathrm{m}$, respectively. ${ }^{*} p<0.05,{ }^{* *} p<0.01, * * * p<0.001$.

\section{Figure 5 The image analysis showed that FA primed SSCs combined with microcryogel promotes bone repair in a murine irradiated bone defect model}

FA-treated irradiated SSCs or irradiated SSCs were combined with a microniche to develop an injectable SSC-microcryogel (Figure 5A). As shown in Figure 5B, more newborn bone was observed in the irradiated-SSC-combined-with-microcryogel group than in the negative control group and the microcryogel group by micro-CT screening at 1, 2, and 3 weeks after SSC microcryogel transplantation. Transplantation of FA-treated irradiated SSCs combined with microcryogel yielded strengthened bone formation in the defect compared with its counterpart without FA treatment (Figure 5B). In addition, BV/TV was higher in the irradiated SSCmicrocryogel group than in the negative control group and microcryogel group. The FA-treated irradiated SSC microcryogel group showed a greater increase in BV/TV than the irradiated 
SSC-microcryogel group (Figure 5C) $\left(* * p<0.01,{ }^{* * *} p<0.001\right)$.

Figure 6 The pathological analysis of FA primed SSCs combined with microcryogel promotes bone repair in a murine irradiated bone defect model

The histological data showed that only fibrous-like connective tissues filled the defect even at week 3 engrafted with microcryogel. In addition, small bone nodules were observed in the bone defect transplanted with the irradiated SSC microcryogel (Figure 6A and 6B). Notably, bonelike tissues filled most of the defects engrafted with FA-treated irradiated SSC microcryogels. The results of Masson's trichrome, OCN, and Col-I staining further validated that FA promoted bone regeneration in defects (Figure 6A and 6B). The quantitative analysis of new bone was further performed according to the results of HE and Masson's trichrome staining. The femur defect repair percentage was calculated by Image-Pro Plus. Figure 6C shows that new bone formation in the defect engrafted by FA-treated irradiated SSC microcryogels $(28.3 \pm 1.1 \%$ at day $7,32.6 \pm 1.9 \%$ at day $14,38.6 \pm 2.1 \%$ at day 21 ) was higher than in defect grafts engrafted by irradiated SSC microcryogels $(21.6 \pm 2.1 \%$ at day $7,24.0 \pm 2.9 \%$ at day $14,28.9 \pm 3.2 \%$ at day 21) Bars in Figure 6A and 6B represent $200 \mu \mathrm{m} .{ }^{*}, P<0.05, * *, P<0.01, * * *, P<0.001$.

\section{Figure S1 The influence of irradiation on adipogenic differentiation of SSCs}

No obvious influences of irradiation (2Gy) on adipogenic droplet formation (Figure S1A) and gene expression of adipogenic differentiation of SSC (Figure S1B) were observed in SSCs.

Bars in Figure S1 A represent $200 \mu \mathrm{m}$.

Figure S2 The general change of irradiation-induced gene expression in SSCs 
The data in Figure S2A and S2B present the differentially expressed genes in heatmap and volcano plot format between SSC and irradiated SSC. The results of mRNA sequencing showed that a total of 638 genes were upregulated, and 198 genes were downregulated in the irradiated SSC group compared with the SSC control group (2 Gy, $\mathrm{n}=3$ ).

Figure S3 Blockage of p38/MAPK and ERK/MAPK in SSCs partially abolished FA primed bone regeneration in irradiated bone defects

The imaging results of the bone regeneration of SSCs in each group showed that inactivation of p38/MAPK and ERK/MAPK impaired FA/SSC-mediated structural reconstitution in bone defects (Figure S3A, S3B, and S3C). Further pathological data demonstrated that SB203580 and PD98059 partially abolished the promotive effects of FA on the bone regenerative cap of irradiated SSCs in vivo (Figure S3A, S3B, and S3C). Moreover, blockage of p38/MAPK and ERK/MAPK pathways by specific inhibitors in FA treated irradiated-SSC significant abolished the bone regenerative activities in bone defects $(33.5 \pm 3.6 \%$ vs $19.8 \pm 1.1 \%$ of SB203580, vs $22.1 \pm 0.9 \%$ of PD98059) (Figure S3D). Bars in Figure S3C represent $200 \mu$ m. $* * * p<0.001$. 
A



OGy
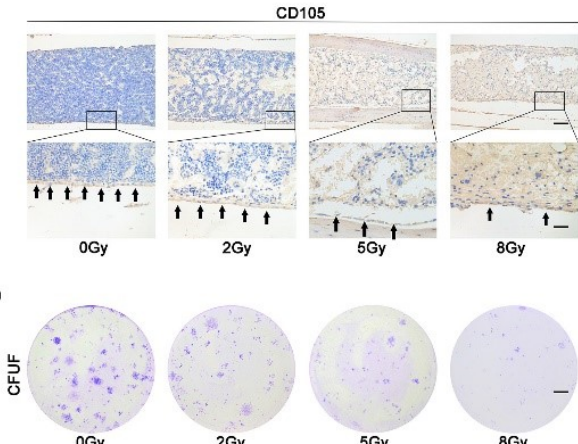

OGy
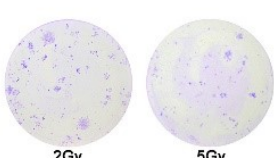

$5 G y$
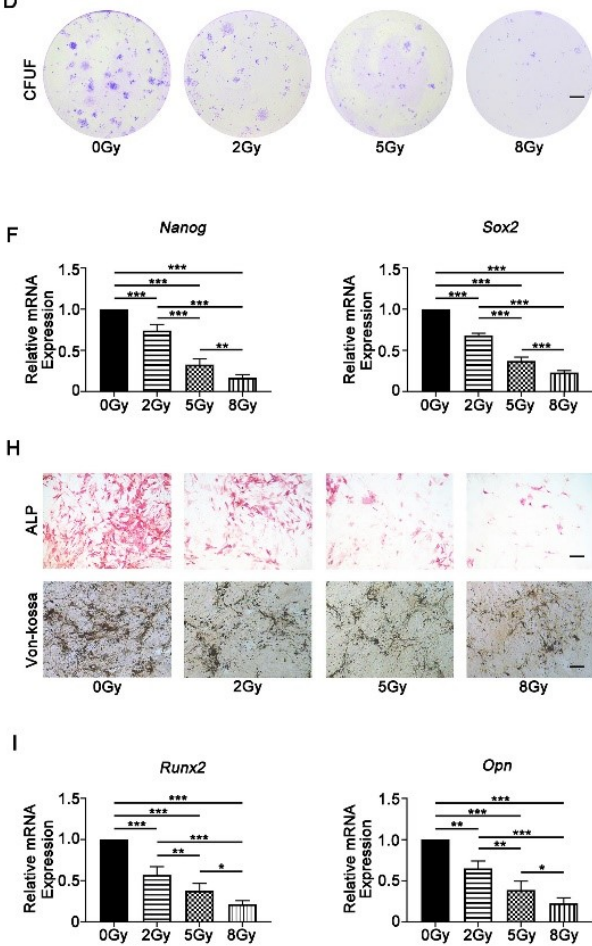

B

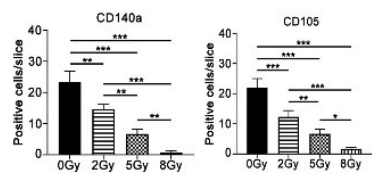

C

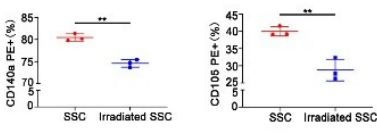

E

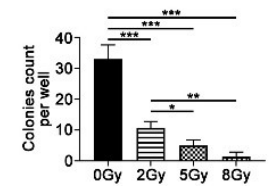

G

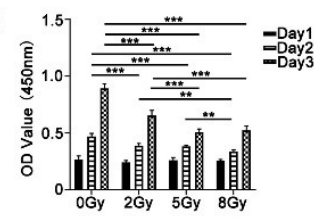


bioRxiv preprint doi: https://doi.org/10.1101/2021.02.14.431131; this version posted February 14, 2021. The copyright holder for this preprint (which was not certified by peer review) is the author/funder. All rights reserved. No reuse allowed without permission.

A
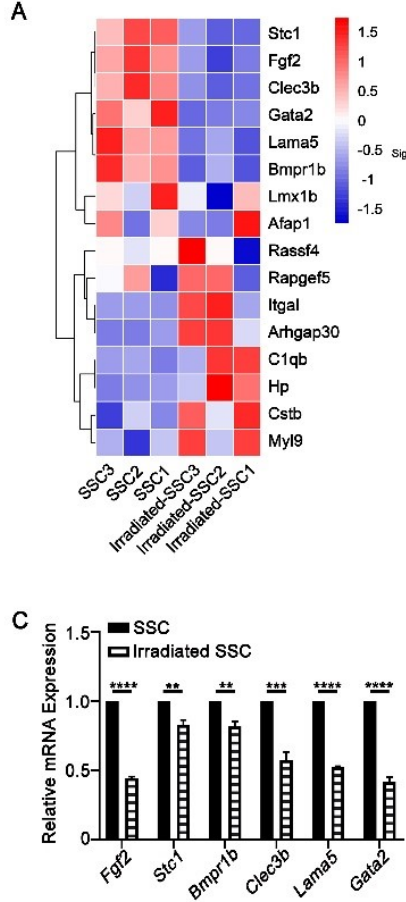

E

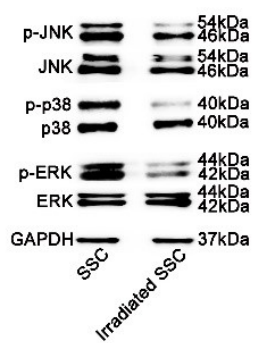

B

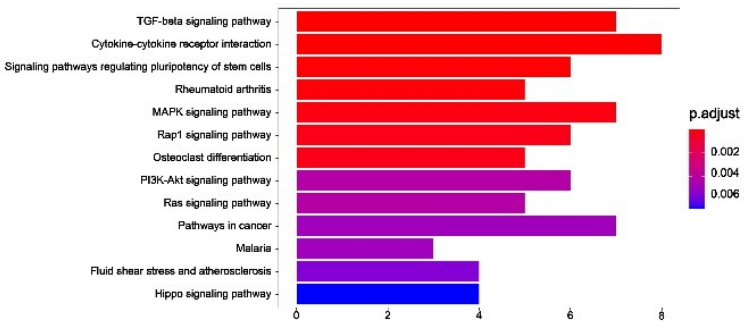

D
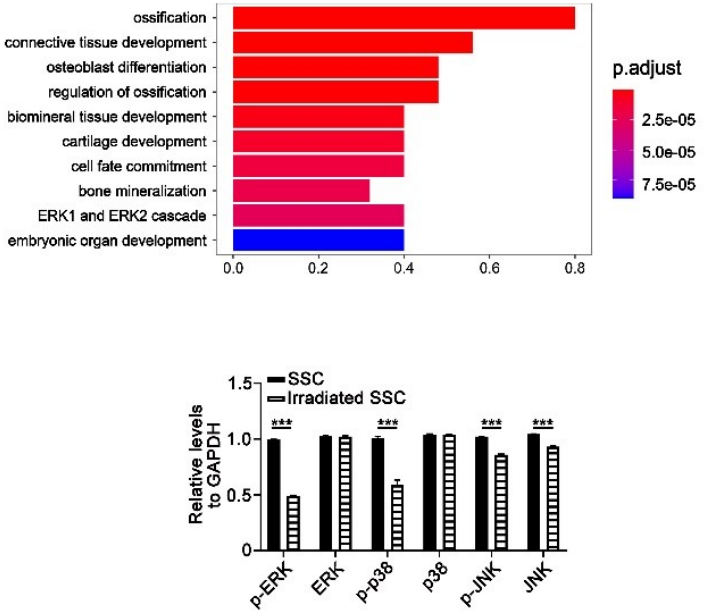
A
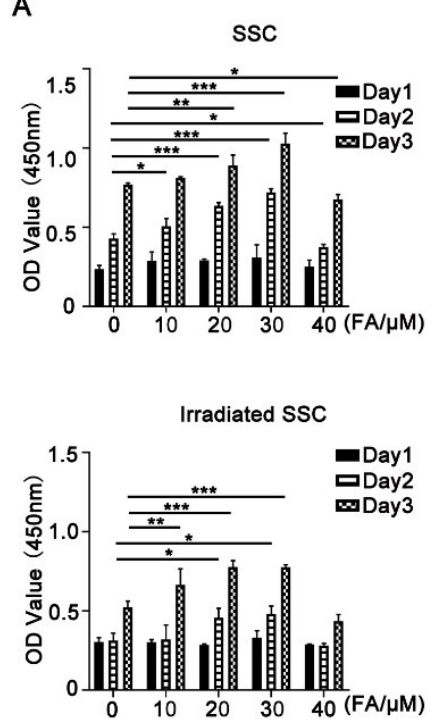

D

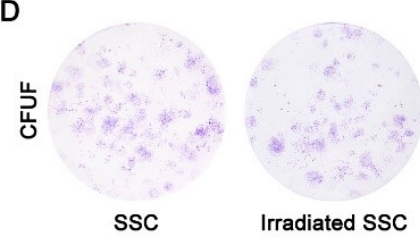

E

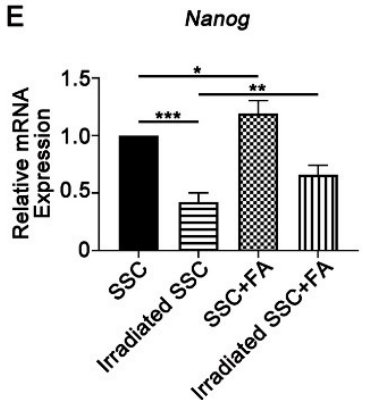

B

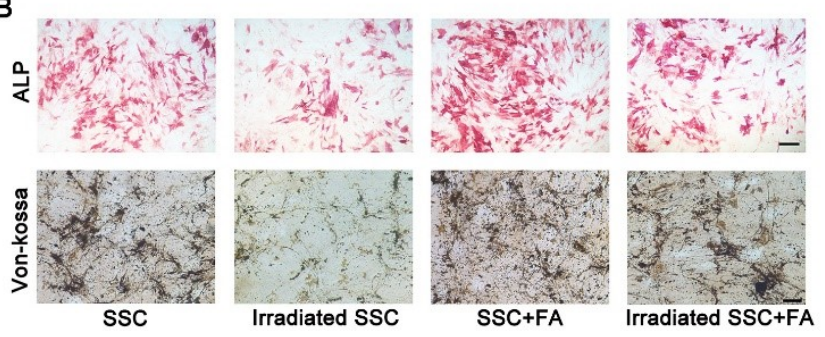

C
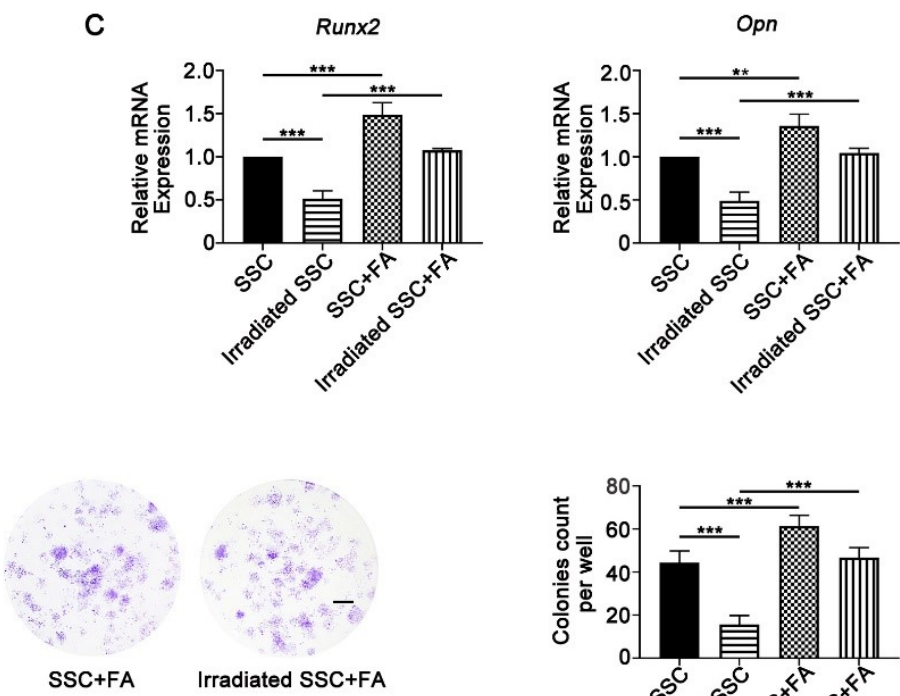

Sox2

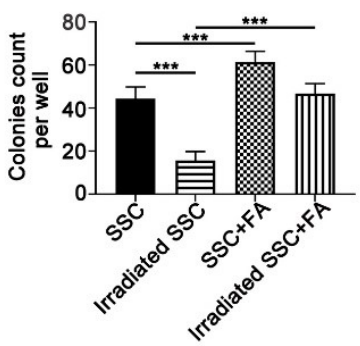

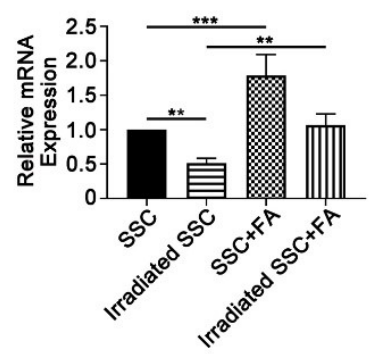


A

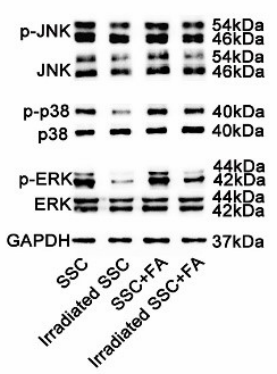

B
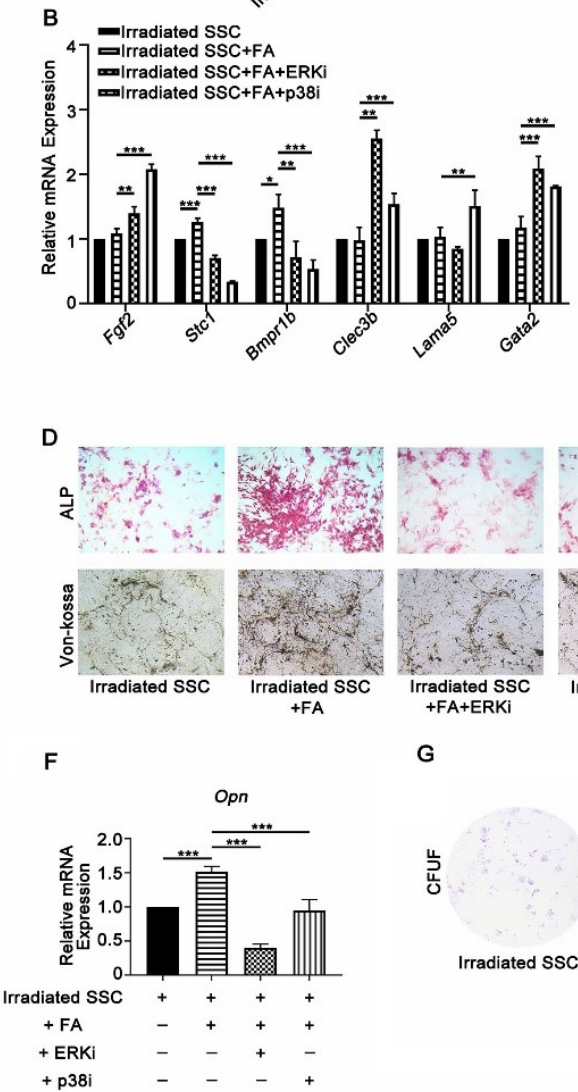

$\mathrm{H}$$$
\text { H }
$$
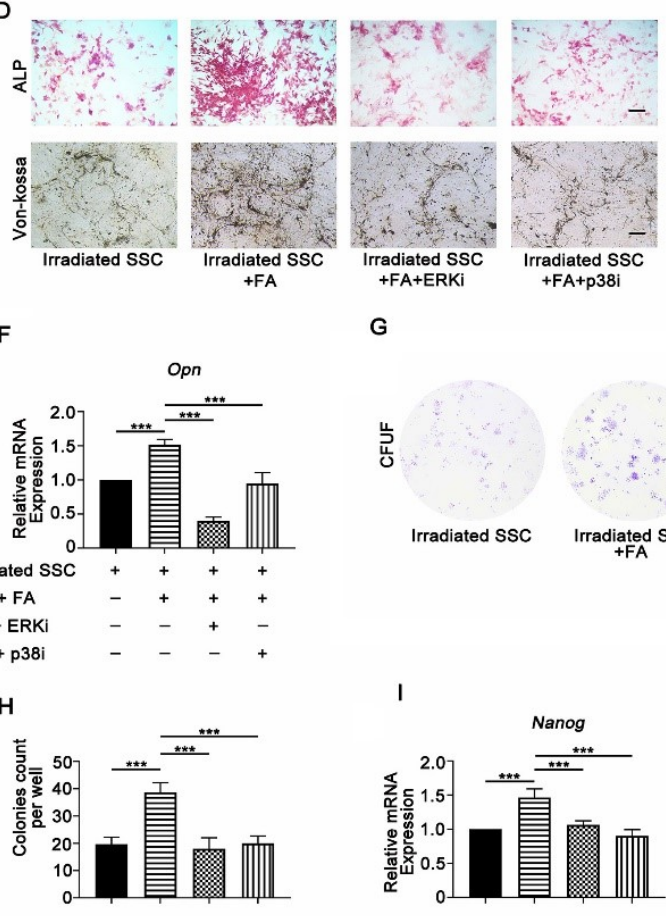

G

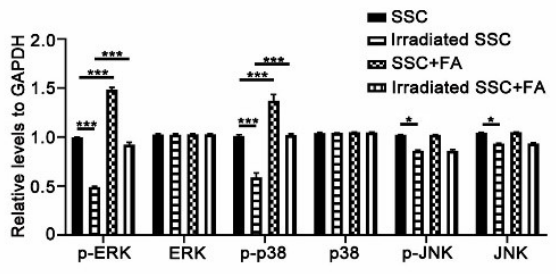

c
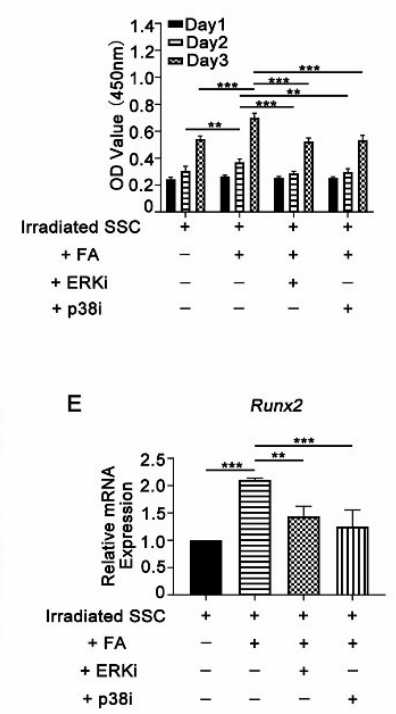

咅
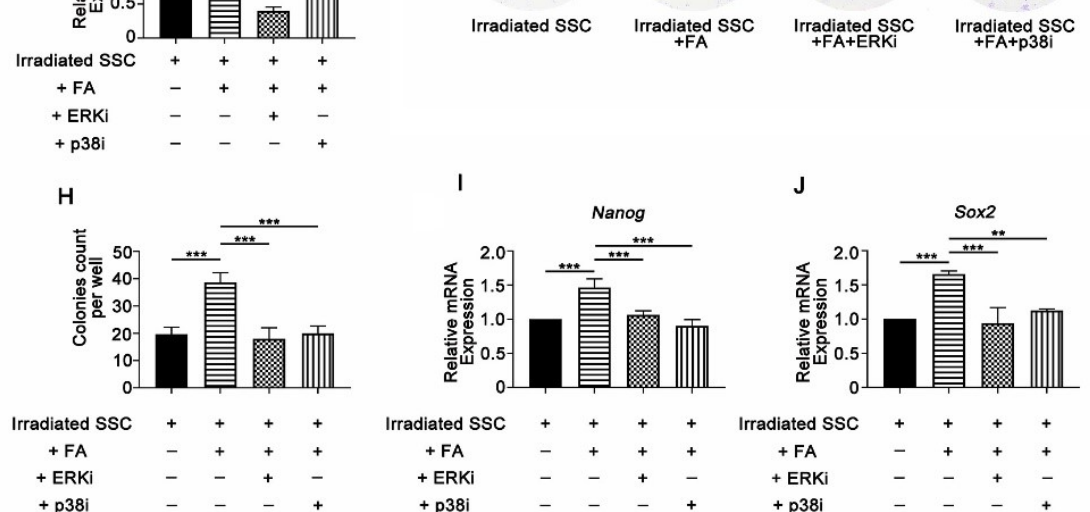

1
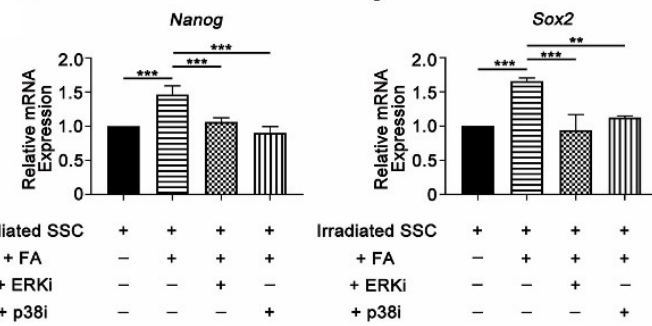
bioRxiv preprint doi: https://doi.org/10.1101/2021.02.14.431131; this version posted February 14, 2021. The copyright holder for this preprint (which was not certified by peer review) is the author/funder. All rights reserved. No reuse allowed without permission.

A
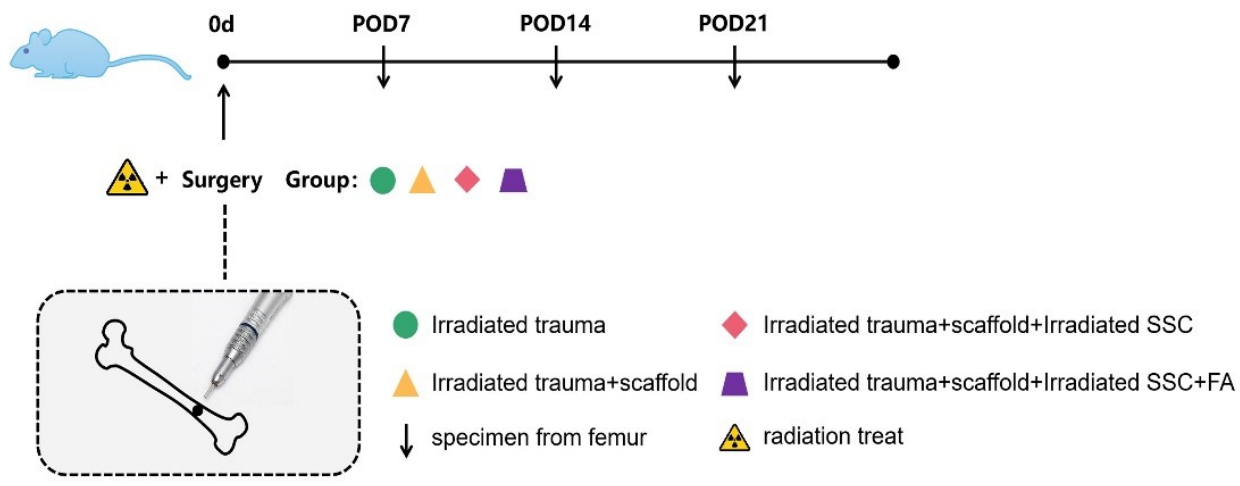

B

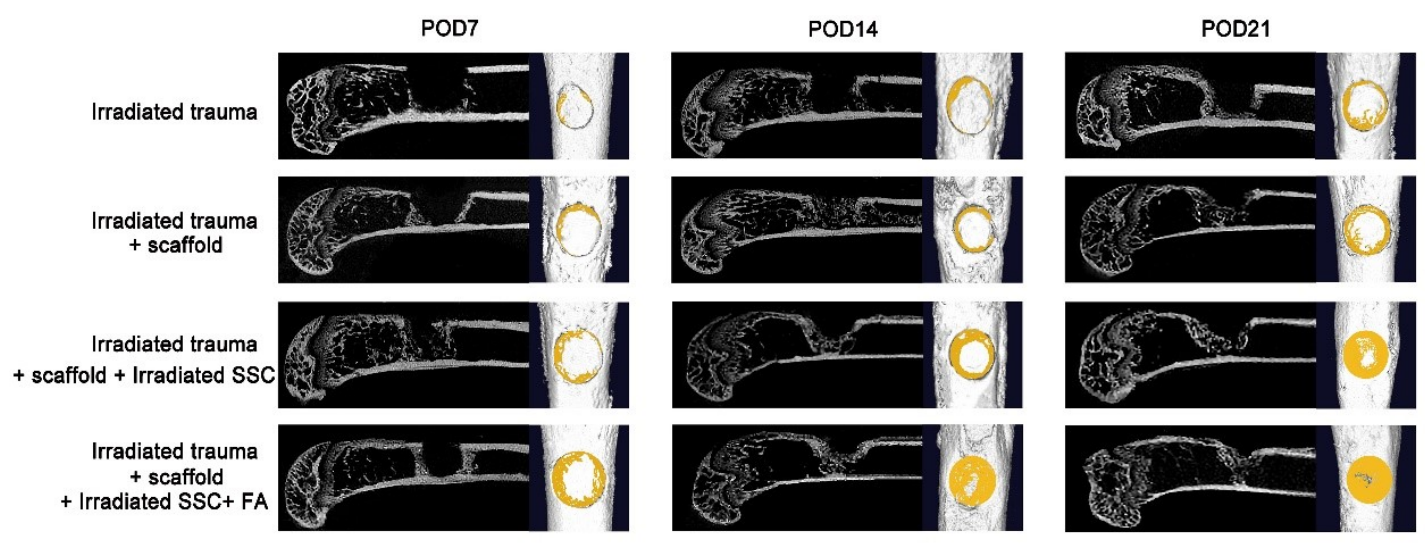

C

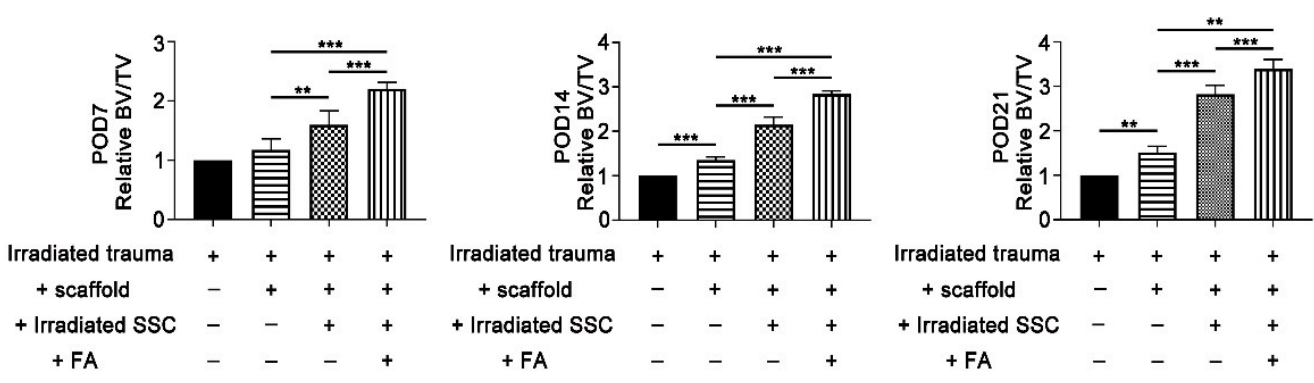


bioRxiv preprint doi: https://doi.org/10.1101/2021.02.14.431131; this version posted February 14, 2021. The copyright holder for this preprint (which was not certified by peer review) is the author/funder. All rights reserved. No reuse allowed without permission.

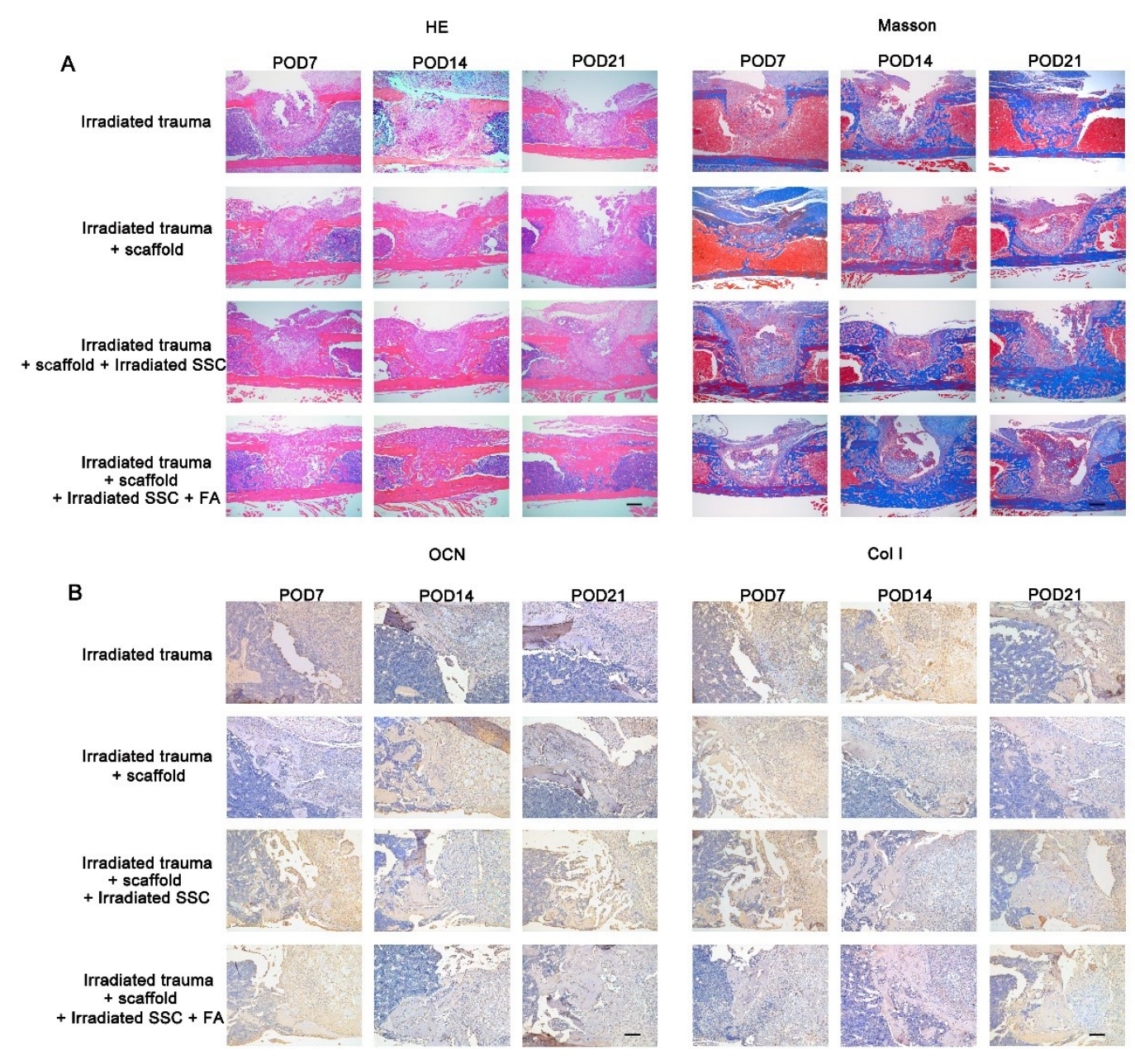

C

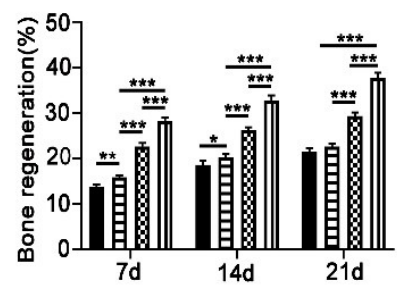

- Irradiated trauma

- Irradiated trauma + scaffold

Irradiated trauma + scaffold + Irradiated SSC

m Irradiated trauma + scaffold + Irradiated SSC + FA 